\title{
Manutenção da permeabilidade dos dispositivos de acesso venoso central em pacientes com câncer
}

\author{
Maintenance of central venous access devices permeability in cancer patients
}

Mantenimiento de la permeabilidad de los dispositivos de acceso venoso central en pacientes con câncer

\author{
Valdemar Franco Cabrera'; Juliana Coutinho de Paula Suguimoto"; Ariane Polidoro Dinilll; \\ Marília Estêvam Cornélio' ${ }^{\text {; }}$ Maria Helena de Melo Limav
}

\begin{abstract}
RESUMO
Objetivo: atualizar o conhecimento sobre a manutenção da permeabilidade dos Dispositivos de Acesso Vascular Central (DAVC) em paciente com câncer. Conteúdo: os pacientes que fazem uso de quimioterápicos muitas vezes necessitam de DAVC seguros e de longa permanência; a obstrução ocorre em $36 \%$ dos pacientes com DAVC inseridos no período de 2 anos, que pode ser classificada como mecânica, por precipitação de medicamento/minerais e pela formação de coágulos. A equipe de enfermagem é responsável por realizar os cuidados para manter a permeabilidade desses dispositivos através do Flushing e Locking. $\mathrm{O}$ uso de heparina demonstrou baixa evidência quando comparado ao Soro Fisiológico (SF), em relação a diminuição da obstrução. Conclusão: o uso de SF na manutenção dos DAVC mostra-se seguro, eficaz e com menor custo financeiro. Descritores: Cateteres venosos centrais; segurança do paciente; neoplasias; permeabilidade.
\end{abstract}

\begin{abstract}
Objective: to update the knowledge on the maintenance of permeability of Central Vascular Access Devices (CVAD) in cancer patients. Content: Patients on chemotherapy often require safe, long-term CKD. Obstruction occurs in 36\% of patients with CVAD inserted in a 2-year period, which can be classified as mechanical due to drug / mineral precipitation and clot formation. The nursing staff is responsible for taking care to maintain the permeability of these devices through Flushing and Locking. The use of heparin showed low evidence when compared to saline (SF), regarding the reduction of obstruction. Conclusion: the use of DES to maintain CVAD is safe, effective and has a lower financial cost.

Descriptors: Central venous catheters; patient safety; neoplasms; permeability.

\section{RESUMEN}

Objetivo: actualizar los conocimientos sobre el mantenimiento de la permeabilidad del dispositivo de acceso vascular central (DAVC) en pacientes con cáncer. Contenido: los pacientes que usan quimioterápicos a menudo necesitan DAVC seguros y de larga permanência. La obstrucción ocurre en el $36 \%$ de los pacientes con DAVC insertados en el período de 2 años y puede ocorir por precipitación de medicamento / minerales y por la formación de coágulos. El equipo de enfermería es responsable de realizar los cuidados para mantener la permeabilidad de estos dispositivos a través del Flushing y Locking. El uso de heparina demostró una baja evidencia en comparación con el Suero Fisiológico (SF), en relación con la disminución de la obstrucción. Conclusión: el uso de SF en el mantenimiento de los DAVC se muestra seguros, eficaz y con menor costo financiero. Descriptores: Catéteres venosos centrales; seguridad del paciente; neoplasias; permeabilidad.
\end{abstract}

\section{INTRODUÇÃO}

Os dispositivos de acesso venoso central (DAVC) são frequentemente confeccionados com silicone ou poliuretano ${ }^{1,2}$. Fornecem acesso venoso seguro para a quimioterapia e contribuem para manutenção da integridade da rede venosa do paciente ${ }^{3}$. Alguns dispositivos são utilizados por longos períodos e necessitam de avaliações frequentes, principalmente em pacientes que utilizam quimioterapia intermitente ${ }^{4}$. Dentre os DAVC mais utilizados no tratamento onco-hematológico estão o Cateter Venoso Central Semi-Implantado, Cateter Central Totalmente Implantado e Cateter Central de Inserção Periférica. A manutenção da permeabilidade destes cateteres é crucial para a qualidade de vida destes pacientes, visto que além do tratamento da quimioterapia este dispositivo é utilizado para coleta de sangue, administração de medicamentos, como analgésicos e antibióticos ${ }^{1,4}$.

'Enfermeiro. Aprimorando em Oncologia e Tratamento antineoplásico. Universidade Estadual de Campinas. São Paulo, Brasil. E-mail: vdmcabrera1@gmail.com "Enfermeira. Ambulatório de Oncologia do Hospital de Clínicas da Unicamp. Mestranda em Assistência ao Paciente Oncológico pela Faculdade de Ciências Médicas. Universidade Estadual de Campinas. São Paulo, Brasil. E-mail: judepaula@hc.unicamp.br

III Enfermeira. Professor Doutor da Área de Administração. Universidade Estadual de Campinas. São Paulo, Brasil. E-mail: adini@unicamp.br

IV Enfermeira. Professor Doutor da Área de Médico-Cirúrgica. Universidade Estadual de Campinas. São Paulo, Brasil. E-mail: mariliacornelio@gmail.com

vEnfermeira. Professora Livre-Docente da Área Fundamental. Universidade Estadual de Campinas. São Paulo, Brasil. E-mail: melolima@unicamp.br 
De acordo com a literatura, a manutenção da permeabilidade dos DAVC pode ser realizada com heparina ou cloreto de sódio $0,9 \%(S F)^{3,5,6}$. Entretanto, a longa permanência aumenta a incidência de complicações locais, tais como a obstrução do lúmen do cateter, o que acarreta a necessidade da troca, substituição ou retirada, ocasionando a interrupção do tratamento, comorbidades e aumento do custo para o serviço de saúde ${ }^{7}$. A obstrução de cateteres em pacientes adultos ocorre em 36\% dos DAVC com 2 anos de inserção ${ }^{8}$.

Obstrução do cateter pode ser definido como oclusão parcial, ou seja, não há retorno de sangue, mas ocorre à infusão de fluidos. Sendo que na oclusão completa não ocorre infusão de fluidos ou retorno venoso. Os fatores relacionados à oclusão do lúmen do cateter podem ser classificados em três categorias: disfunção mecânico, precipitação de medicamentos/minerais e a formação de coágulo,10. O mal posicionamento da ponta do cateter, extensões dobradas e fixação inadequada levam a oclusão mecânica. Em relação a precipitação dos medicamentos podem-se formar precipitados dentro do lúmen após a administração de minerais, lipídios ou por medicamentos incompatíveis. A oclusão trombótica está relacionada a presença de fibrina e os constituintes do sangue que acumulam dentro do lúmen ou em torno da extremidade do cateter e o vaso ${ }^{11}$. As oclusões trombóticas são mais severas do que as demais, pois aumentam o risco de trombose vascular relacionada ao cateter. Além disso, a fibrina favorece o crescimento de bactéria e infecção ${ }^{12,13}$.

Diante da relevância da manutenção da permeabilidade de dispositivos de acesso venoso central na qualidade de vida durante o tratamento de câncer, este trabalho teve o objetivo de atualizar o conhecimento sobre a manutenção da permeabilidade dos dispositivos de acesso vascular central (DAVC) em paciente com câncer.

\section{CUIDADOS NA MANUTENÇÃO DA PERMEABILIDADE DOS DAVC}

$\mathrm{Na}$ atualidade a inserção e os cuidados com DAVC tem participação de uma equipe multiprofissional, envolvendo enfermeiros, médicos oncologistas, hematologistas, radiologistas, cirurgiões, infectologistas, e em algumas instituições existe a presença de times de Terapia Intravenosa ${ }^{14,15}$. Por outro lado, os cuidados com a manutenção da permeabilidade são de responsabilidade da equipe de enfermagem, sendo que o Flushing e o Locking venoso fazem parte dos cuidados padrões para a manutenção e prevenção das complicações dos DAVC de acordo com os guidelines ${ }^{10,16}$.

De acordo com a literatura, o conceito Flushing é a lavagem do cateter, realizado com Soro Fisiológico 0,9\% (SF), antes e após infusão de fluidos, com seringa de $10 \mathrm{ml}$ ou projetadas para produzir baixas pressões. A injeção de SF deve ser realizada em turbilhonamento, com a técnica pulsátil de instilar e pausar entre cada ml no intervalo de tempo de 0,4 segundos e com pressão positiva evitando o refluxo de sangue para o lúmen do cateter. Estudo in vitro verificou que a técnica de turbilhonamento permite melhor retirada do material proteico do lúmen do dispositivo quando comparado com a técnica de infundir SF de maneira contínua 6 6,10,17.

O Locking por sua vez é considerado uma injeção intraluminal, realizado com solução salina ou solução salina com heparina, efetuadas em intervalos de tempo e volume limitado com objetivo de manter o cateter permeável ${ }^{6,10}$. Não existe consenso na literatura em relação à dose adequada para a solução heparinizada, $150 \mathrm{UI} / 3 \mathrm{ml}, 500 \mathrm{UI} / 5 \mathrm{ml}, 1000$ $\mathrm{UI} / 3 \mathrm{ml}$ quando comparado ao SF na manutenção da permeabilidade ${ }^{13,18}$. O intervalo para realização Locking para DAVC é de 4 semanas. Porém estudos têm demonstrando que a extensão deste intervalo para a realização do Locking é eficaz e seguro na manutenção da permeabilidade. Estudo recente corrobora com os anteriores onde não observou diferença entre o curto e o longo período no intervalo do Locking com taxa de complicações quando o cateter está em uso intermitente $\mathrm{e}^{19,20}$.

Estudos de revisão sistemática demonstraram baixa evidência para a superioridade do Locking com heparina quando comparada ao SF na prevenção da oclusão do DAVC. Recentemente uma destas revisões foi reavaliada e observaram que o Locking com heparina intermitente resulta em menor risco para oclusão dos DAVC quando comparado ao SF em adultos. Porém foi mantida a baixa qualidade de evidência para sustentar outra posição da conclusão de $2014^{21}$. Dados recentes demonstraram que o custo médio para manutenção da permeabilidade dos DAVC com heparinização em pacientes em unidades de internação, para nossa realidade brasileira corresponde a US\$ 9.71 (DP=1.35), sendo que com SF é de US\$ 8.81 ( $D P=1.29)$, redução de US\$ 0.90/procedimento22.

\section{MELHORES PRÁTICAS}

O conhecimento atualizado do enfermeiro para os cuidados adequados com os DAVC, a presença de grupos de experts nas instituições de saúde e pacientes bem orientados em relação aos cuidados são fundamentais na prevenção de complicações relacionadas a estes cateteres ${ }^{23-25}$. Para o gerenciamento do cuidado no manejo diário dos DAVC é necessário que a equipe de enfermagem seja estimulada a manter-se atualizada, criar estratégias para a educação continuada, estimular a mudança de comportamento, implementar novas tecnologias e instituir protocolos que possam 
auxiliar procedimentos baseado em evidências científicas ${ }^{26-28}$, visto que dados do Instituto do Câncer estima 420 mil novos caso de câncer entre 2018-2019²9.

\section{CONCLUSÃO}

O tratamento na maioria das vezes demandará o uso de quimioterápicos com DAVC, com uma assistência segura e promoção da qualidade de vida. A oclusão é uma complicação que pode ser reduzida com a implementação de protocolos institucionais de Flushing e Locking. Diante das baixas evidências em relação ao uso de heparina ser superior ao SF na prevenção da oclusão do lúmen dos cateteres, e com conhecimento dos benefícios do SF para manutenção da permeabilidade, juntamente com as recentes informações sobre os custos de manutenção destes cateteres é possível considerar que o uso do SF é seguro e eficaz e traz menor custo financeiro para manutenção dos DAVC em uso intermitente.

\section{REFERÊNCIAS}

1. Agência Nacional de Vigilância Sanitária (Br). Medidas de prevenção de infecção relacionada à assistência à saúde. Brasília (DF): Anvisa. [Internet], 2017[cited 2019 Nov 29]. Available from:

http://portal.anvisa.gov.br/documents/33852/3507912/Caderno+4+-

+Medidas+de+Preven\%C3\%A7\%C3\%A30+de+Infec\%C3\%A7\%C3\%A3o+Relacionada+\%C3\%A0+Assist\%C3\%AAncia+\%C3\%A0+Sa\% C3\%BAde/a3f23dfb-2c54-4e64-881c-fccf9220c373

2. Cohen AB, Dagli M, Stavropoulos SW, Mondschein JI, Soulen MC, Shlansky-Goldberg RD, et al. Silicone and polyurethane tunneled infusion catheters: A comparison of durability and breakage rates. J Vasc Interv Radiol. [Internet], 2011 [cited 01 dez 2018]. 22:638-41. DOI: http://dx.doi.org/10.1016/j.jvir.2011.01.433

3. Schiffer CA, Mangu PB, Wade JC, Camp-Sorrell D, Cope DG, El-Rayes BF, et al. Central venous catheter care for the patient with cancer: American Society of Clinical Oncology Clinical Practice Guideline. J Clin Oncol. [Internet], 2013 [cited 28 Nov 2019]; 31(10):1357-370. DOI: https://doi.org/10.1200/JCO.2012.45.5733

4. Couban S, Goodyear M, Burnell M, Dolan S, Wasi P, Barnes D, et al. Randomized placebo-controlled study of low-dose warfarin for the prevention of central venous catheter-associated thrombosis in patients with cancer. J Clin Oncol. [Internet], 2005[cited 2019 Nov 29]; 23(18): 4063-9. DOI: https://doi.org/10.1200/JCO.2005.10.192

5. Santos EJF, Nunes MMJ, Cardoso DFB, Apóstolo JLA, Queirós PJP, Rodrigues MA. Effectiveness of heparin versus $0.9 \%$ saline solution in maintaining the permeability of central venous catheters: A systematic review. Rev. Esc. Enferm. USP. [Internet], 2015 [cited 2019 Nov 29]; 49(6): 995-1003. DOI: http://dx.doi.org/10.1590/S0080-623420150000600017

6. Goossens GA. Flushing and locking of venous catheters: Available evidence and evidence deficit. Nurs Res Pract. [Internet], 2015 [cited 2019 Nov 29]; 985686. DOI: https://doi.org/10.1155/2015/985686

7. Zhong L, Wang HL, Xu B, Yuan Y, Wang $X$, Zhang Y, et al. Normal saline versus heparin for patency of central venous catheters in adult patients- a systematic review and meta-analysis. Crit Care. [Internet], 2017 [cited 2019 Nov 30]; 21:5. DOI: https://doi.org/10.1186/s13054-016-1585-x

8. López-Briz E, Ruiz Garcia V, Cabello JB, Bort-Martí S, Carbonell Sanchis R, Burls A. Heparin versus $0.9 \%$ sodium chloride locking for prevention of occlusion in central venous catheters in adults. Cochrane Database Syst Rev. [Internet], 2018 [cited 2019 Nov 30];7:CD008462. DOI: https://doi.org/10.1002/14651858.CD008462.pub3

9. Dal Molin A, Clerico M, Baccini M, Guerretta L, Sartorello B, Rasero L. Normal saline versus heparin solution to lock totally implanted venous access devices: Results from a multicenter randomized trial. Eur J Oncol Nurs. [Internet], 2015; [cited 2019 Nov 30];19: 638-43. DOI: https://doi.org/10.1016/j.ejon.2015.04.001

10. Brito ARO, Nishinari K, Saad PF, Saad KR, Pereira MAT, Emídio SCD, et al. Comparison between saline solution containing heparin versus saline solution in the lock of totally implantable catheters. Ann Vasc Surg. [Internet], 2018; [cited 2019 Nov 30]; 47:85-9. Available from: https://www.annalsofvascularsurgery.com/article/S0890-5096(17)31011-7/fulltext

11. Goossens GA, Jérôme $M$, Janssens $C$, Peetermans WE, Fieuws $S$, Moons $P$, et al. Comparing normal saline versus diluted heparin to lock non-valved totally implantable venous access devices in cancer patients: A randomised, non-inferiority, open trial. Ann Oncol. [Internet], 2013; [cited 2019 Nov 30]; 24:1892-9. DOI: https://doi.org/10.1093/annonc/mdt114

12. Pittiruti M, Bertoglio S, Scoppettuolo G, Biffi R, Lamperti M, Dal Molin A, et al. Evidence-based criteria for the choice and the clinical use of the most appropriate lock solutions for central venous catheters (Excluding dialysis catheters): A GAVeCeLT consensus. J Vasc Access. [internet]. 2016; [cited 2019 Nov 30]; 17: 453-64. DOI: https://doi.org/10.5301/jva.5000576

13. Mitchell MD, Anderson BJ, Williams K, Umscheid CA. Heparin flushing and other interventions to maintain patency of central venous catheters: A systematic review. J Adv Nurs. [Internet], 2009 [cited 2019 Nov 29]; 65(10):2007-21. DOI: https://doi.org/10.1111/j.1365-2648.2009.05103.x

14. Baskin JL, Pui CH, Reiss U, Wilimas JA, Metzger ML, Ribeiro RC, et al. Management of occlusion and thrombosis associated with long-term indwelling central venous catheters. Lancet. [Internet], 2009 [cited 2019 Nov 29]; 374(9684): 159-169. DOI: https://doi.org/10.1016/S0140-6736(09)60220-8

15. Van Miert C, Hill R, Jones L. Interventions for restoring patency of occluded central venous catheter lumens (Review). Cochrane Database Syst Rev. [internet], 2012 [cited 2019 Nov 29]; (4): CD007119. DOI:

https://doi.org/10.1002/14651858.CD007119.pub2 
16. Lee AY, Levine MN, Butler G, Webb C, Costantini L, Gu C, et al. Incidence, risk factors, and outcomes of catheter-related thrombosis in adult patients with cancer. J Clin Oncol. [internet], 2006 [cited2019 Nov 30]; 24:1404-8. Available from: https://ascopubs.org/doi/10.1200/JCO.2005.03.5600

17. Timsit JF, Farkas JC, Boyer JM, Martin JB, Misset B, Renaud B, et al. Central vein catheter-related thrombosis in intensive care patients: Incidence, risks factors, and relationship with catheter-related sepsis. Chest. [Internet], 1998 [cited 2019 Nov 29]; 114(1):207-13. DOI: https://doi.org/10.1378/chest.114.1.207

18. Kahale LA, Tsolakian IG, Hakoum MB, Matar CF, Barba M, Yosuico VE, et al. Anticoagulation for people with cancer and central venous catheters. Cochrane Database Syst Rev. [Internet], 2018 [cited 2019 Nov 30]; 6:CD006468. DOI: https://doi.org/10.1002/14651858.CD006468.pub6

19. Teichgräber UK, Pfitzmann R, Hofmann HAF. Central venous port systems as an integral part of chemotherapy. Dtsch Arztebl Int. [Internet], 2011 [cited 2019 Nov 29]; 108(9):147-53. DOI: https://doi.org/10.3238/arztebl.2011.0147

20. Rasero L, Golin L, Ditta S, Massimo DS Di, Molin AD, Piemonte G. Effects of prolonged flushing interval in totally implantable venous access devices (TIVADs). Br J Nurs. [Internet], 2018 [cited 2019 Nov 29]; 27(8):s4-s10. DOI: https://doi.org/10.12968/bjon.2018.27.8.S4

21. Solinas G, Platini F, Trivellato M, Rigo C, Alabiso O, Galetto AS. Port in oncology practice: 3-monthly locking with normal saline for catheter maintenance, a preliminary report. J Vasc Access. [Internet], 2017 [cited 2019 Nov 29];18(4):325-327. DOI: https://doi.org/10.5301/jva.5000740

22. Homo RFB, Lima AFC. Direct cost of maintenance of totally implanted central venous catheter patency. Rev. latinoam. enferm. [Internet], 2018 [cited 2019 Nov 29]; 26: e3004. DOI: https://doi.org/10.1590/1518-8345.2263.3004

23. Karamanoglu A, Yumuk PF, Gumus M, Ekenel M, Aliustaoglu M, Selimen D, et al. Port Needles: Do They need to be removed as frequently in infusional chemotherapy? J Infus Nurs. [Inerrnet], 2003 [cited 2019 Nov 29];26(4):239-42. DOI: https://doi.org/10.1097/00129804-200307000-00009

24. Sá Neto JA, Silva ACSS, Vidal AR, Knupp VMAOK, Barcia LLC, Barreto ACM. Nurses' knowledge of the peripherally inserted central catheter: local realities and global challenges. Rev. enferm. UERJ. [Internet], 2018 [cited 2019 Nov 29]; 26:e33181. DOI: http://dx.doi.org/10.12957/reuerj.2018.33181

25. Almeida TM, Gallasch CH, Gomes HF, Fonseca BO, Pires AS, Peres EM. Prevention of infection relating to the short-term, nonimplanted, central-line catheter. Rev. enferm. UERJ. [Internet], 2018 [cited 2019 Nov 29]; 26: e31771. DOI: http://dx.doi.org/10.12957/reuerj.2018.31771

26. Davis D, Bordage G, Moores LK, Bennett N, Marinopoulos SS, Mazmanian PE, et al. The science of continuing medical education: Terms, tools, and gaps. Chest. [Internet], 2009 [cited 2019 Nov 29]; 135(3): 8S-16S. DOI: https://doi.org/10.1378/chest.08-2513

27. Sichieri K, lida LIS, De Menezes IRDSC, Carlos Garcia P, Santos TR, Peres E, et al. Central line bundle maintenance among adults in a university hospital intensive care unit in São Paulo, Brazil: A best practice implementation project. JBI Database System. Rev. Implement. Rep. [Internet], 2018 [cited 2019 Nov 29]; 16(6):1454-73. DOI: https://doi.org/10.11124/JBISRIR-2017-003561

28. Registered Nurses' Association of Ontario. Care and Maintenance to Reduce Vascular Access Complications. [internet], 2008 [cited 2019 Dec 01]; Available from https://rnao.ca/bpg/guidelines/care-and-maintenance-reduce-vascular-accesscomplications.pdf

29. Instituto Nacional de Câncer José Alencar Gomes da Silva (Br). Estimativa 2018: incidência de câncer no Brasil. Coordenação de Prevenção e Vigilância. Rio de Janeiro (RJ): INCA. [Internet], 2017 [cited 2019 Nov 29]. https://doi.org/10.32635/21769745.RBC.2018v64n1.115 